\title{
Alternative reproductive strategies in wasps: queen, worker, or both?
}

\author{
M. H. Richards ${ }^{1}$
}

Published online: 1 July 2019

(c) International Union for the Study of Social Insects (IUSSI) 2019

It is practically axiomatic in animal behaviour and evolution textbooks that in social insects, a helper achieves greater inclusive fitness through raising non-descendant kin than by raising her own offspring. If workers are assumed to be sterile, then their direct fitness is zero, and the adaptive value of helping behaviour can be explained solely through indirect fitness gains. However, in many social insects, it is inaccurate to generally characterize workers as sterile. There are numerous examples of social insect workers or subordinates that lay eggs and produce their own brood. This means that we cannot ignore workers' potential for achieving some measure of direct fitness, in addition to whatever indirect fitness they accrue through helping to raise relatives. In some species, especially those usually referred to as primitively eusocial, females are totipotent at eclosion, so have the flexibility to become egg layers, helpers, or both, switching roles according to current social and ecological constraints. Species with totipotent females are especially likely to provide evidence that sometime subordinates mix indirect and direct reproductive tactics that optimize lifetime inclusive fitness.

In this issue, Brahma et al. (2019) examine reproductive flexibility and the dynamics of colony initiation in Ropalidia marginata, a tropical vespid wasp with year-round colony initiation. Like many vespids, $R$. marginata builds open nests without an envelope, which means that biologists can watch nest inhabitants interacting with each other and with brood. Nests are founded either by a single foundress or by a small group of females, only one of which becomes the egglaying queen, while the others become non-laying workers (Gadagkar 2001). Colonies grow as female brood eclose and remain on the natal nest as workers. However, observations of wild colonies demonstrate that after eclosion, females that seem destined to become workers can avail themselves of other opportunities, by leaving their natal nest to join other nests or to initiate their own nests. Not only do newly

M. H. Richards

mrichards@brocku.ca

1 Brock University, St. Catharines, ON, Canada eclosed females have a variety of reproductive options to choose from, but $R$. marginata females live long enough that over the course of a lifetime, they may fill more than one reproductive role.

To understand how wasps balance opportunities to gain direct vs. indirect fitness, it is necessary to quantify how often females choose to remain on their natal nests as workers, join other nests, or initiate new nests. As anyone who has tried to follow insects in field conditions will surmise, following large numbers of wasps from their natal nests to new nest sites would be practically impossible to do in the wild. Brahma et al. take advantage of a useful characteristic of $R$. marginata, which is their willingness to nest in large walk-in cages that allow biologists to observe the processes and behaviours involved in colony initiation. Their experiment began when 9 large parent colonies (median size 64 adult females and $>100$ brood) were brought into the cages. Over the course of 5-6 months, females from these parental colonies founded a total of 29 new nests ( 9 single foundress and 20 multi-foundress), all of which successfully produced offspring that eclosed before the experiment was terminated. Most females $(65 \%)$ remained in their natal colonies as workers, $23 \%$ joined different colonies, and $12 \%$ became foundresses of new nests.

Some fascinating behaviours were observed as female wasps adopted different roles and chose where to live. Females that initiated the 20 new multi-foundress nests, first formed off-nest assemblies in which they engaged in dominance interactions, which also determined which female would become the egg-laying queen in the new colony. Females that became single foundresses did not participate in these assemblies. This contrast suggests that females decided whether to become single or cofoundresses prior to leaving their natal nests. The multi-foundress assemblies of $R$. marginata sound almost like a lek in which females display to each other in a symbolic space, before proceeding to the actual space in which they will build a nest.

The behavioral flexibility demonstrated by $R$. marginata females in this study implies that these wasps continually assess their current reproductive status in relation to future 
reproductive status and adjust their behavior in a way that optimizes their lifetime reproductive success. I was particularly convinced of this by the observation of nine cofoundresses of new nests that became "drifters" that continued to bring food to their parent colonies, as well as to their new colonies. One drifter eventually returned to her parent colony where she ultimately became the queen.

The ability to assess and trade off current versus future reproductive effort has now been observed in at least three different vespid wasps living in environments with long breeding seasons (see Cant and Field 2001, Leadbeater et al. 2011). I suspect that in social insects with long-lived, totipotent females, we will find more examples in which individuals optimize lifetime inclusive fitness through tradeoffs between alloparental and parental reproduction.

\section{References}

Brahma A, Mandal S, Gadagkar R (2019) To leave or to stay: direct fitness through natural nest foundation in a primitively eusocial wasp. Insectes Soc. https://doi.org/10.1007/s00040-019-00702-2

Cant MA, Field J (2001) Helping effort and future fitness in cooperative animal societies. Proc R Soc Lond Ser B Biol Sci 268:1959-1964

Gadagkar R (2001) The social biology of Ropalidia marginata: toward understanding the evolution of eusociality. Harvard University Press, Cambridge

Leadbeater E, Carruthers JM, Green JP et al (2011) Nest inheritance is the missing source of direct fitness in a primitively eusocial insect. Science 333:874-876 